\title{
A Novel Approach for Iceberg Query Evaluation on Multiple Attributes Using Set Representation
}

\author{
V. Chandra Shekhar Rao ${ }^{1}$, Dr. P. Sammulal ${ }^{2}$, \\ ${ }^{1}$ Research Scalar, Department of CSE, JNTU, Hyderabad, vcsrao.kitswgl@gmail.com \\ ${ }^{2}$ Associate Professor, Department of CSE, JNTU, Hyderabad, sammulalporika@g
}

\section{Article Info \\ Article history: \\ Received Jun $12^{\text {th }}, 2018$ \\ Revised Aug 20 ${ }^{\text {th }}, 2018$ \\ Accepted Aug 26 $6^{\text {th }}, 2018$}

\section{Keyword:}

set operations

iceberg query

Database

threshold

\begin{abstract}
Iceberg query (IBQ) can be an really identifying kind of aggregation question that calculate aggregations up-on user given threshold (T). In data mining field, effective investigation of compounding queries was because of by the majority of investigators because the tremendous generation of information outside of industrial and businesses industries. Conclusion assist database and discovery of the majority of information connected systems largely calculate the worthiness of most fascinating features having an critical level of information from data foundations that may be tremendous. By means of the paper, we propose that an initial Manner of calculating IBQ, which builds a choice for every attribute nicely value, but additionally includes a One of a Kind events Inside the attribute column also plays specify operations for creating closing Outcomes. We formulated highly effective GUI software for just 2 characteristics, numerous traits employing egotistical prepare and several features utilizing lively plan. If data collection comprises two traits, then it truly is substantially more advanced than apply just two traits. In the event of information collection comprises multiple traits, predicated up on anyone choice suitable module could potentially be decided on. If characteristic uniqueness changes from characteristic in to the following characteristic, then vibrant variety approach is very powerful. This strategy somewhat reduces performance memory and time space contrast with additional processes. A experiment using artificial Statistics collection and actual info demonstrates our strategy will be considerably more effective compared to present apps for Nearly Every threshold.
\end{abstract}

\section{Corresponding Author:}

Dr. P. Sammulal,

Associate Professor, Department of CSE, JNTU,

Hyderabad, sammulalporika@gmail.com

\section{Introduction}

Industry awareness and Recognizing Detection [1] from Dealing databases/warehouses always hardy firearms, So that you may acquire competitive wages in the current industry community. An Iceberg Query(IBQ) could be an excellent Form of a aggregation question that divides values to a Individual Outlined threshold (T) meanings. It truly is of these differentiating comprehension of most those end users in bringing advanced level degree worth which frequently take more considerable in manufacturing companies. The Syntax with the Iceberg query as to a romantic relationship REL $(\mathrm{C} 1, \mathrm{C} 2 \ldots \mathrm{Cn})$ is revealed beneath:

SELECT Ci, Cj, .., Cm, AGG(*) FROM R GROUP BY Ti,Tj..., Tm HAVING AGG $(*)>=$ T

This aggregation performs,"at which by $\mathrm{Ci}, \mathrm{Cj}, \ldots, \mathrm{Tm}$ " suggests a sub set pair of capacities in well-known mix faculties. Aggregation functions just like COUNT(),COUNT(*),MIN,MAX,SUM and AVG. The more 
expensive in comparison with equal to $>=$ could possibly be considered a index used like a contrast predicate.

1.1 Market Basket Analysis Marketplace analysts apply market place basket issues into substantial data warehouses [2] that save purchaser earnings transactions. These inquiries explain person paying for patterns, so thus by delivering thing monies (and triples) that may be attracted collectively by numerous clients. Assessing those questions will be powered with huge numbers collections. We utilize exactly the Business basket question locate normally happening term monies.

1.2 Set Operations Advantages Set up operations greatly help speed the Iceberg Queries in diminished execution period in contrast with remaining IBQ techniques like tuple scan-based prepare and lively pruning. It lowers the amount of iterations amongst put pairs of 2 exceptional faculties by occurring difference so correcting the sets that doesn't fit the ceremony worth. Moreover, it helps in repairing the locations thus decreasing the iterations Following the threshold ailment does not match.

When applications can be hard to get the job done with, it compels users to-do glitches, or no matter whether it frees users attempts to achieve their particular objectives, subsequently can hate that, irrespective of computational potential it displays or even the functionality it includes; since it ultimately ends up making a customer's perception of these applications, or so the interface must be proper. The minute we appear programs, we have to contemplate the future customers, such as profiles with the age, instruction, gender, physical talents and cultural or cultural heritage, enthusiasm, goals and disposition. For the explanation, one port design may possibly perhaps not be perfect for a number of computer system users although it might possibly be just precious to specified users.

\section{Related work}

A number of this plan that may be helpful for smaller sized database would be: Sorting REL on disc afterward proceeds aggregating and selecting the formerly recorded threshold values. These procedures don't scale into large information collections. Thus, other procedures are indispensable. A couple Them have been:

\subsection{Sampling}

This System samples a few of Documents by Your Bond, aggregates and extracts Which the Documents "Candidates in to the Prior Remedy" Which (the sample size) Go the threshold.

\subsection{Bucket counting}

As opposed to committing a counter to every single various selling price, give a counter tops for a pair of special worth, acquiring a hash functionality to split the worth into classes. These cubes create bogus favorable, values which can be thought candidates to this prior remedy but usually do not transcend this threshold.

\subsection{Tuple scan based approach}

More of the query advertising techniques for calculating compilation queries could be categorized whilst the tuple scanning base application, which requires the minimum minimal of 1 definite dining table scanning to navigate advice out-of disc drive. They pay give attention to lessening the reach of motions that the instant the data dimensions is not important. None has effectively mastered the territory of compounding concerns to Come Across powerful communication. This kind of tuple-scan-based strategy frequently wants a very long time to respond unanswered queries, particularly in the event your dining table remains nonetheless quite important. Besides those tuple-scan-based strategies, manufactured a two-level hardly any map index that could potentially be leveraged processing compilation queries.

You'll come across several exceptional information structures employed in analytics base to receive paid indexes used to rapidly evaluate queries. Truly one of these basic kinds of signs is termed as a bit map 
indicator. Little map indices are shown to triumph [6], notably due to analysis mainly or append just data, and in addition are normally utilized at the information warehousing software and pillar stores applying little map indices, we want must attain little map indices in their aggregate faculties. Second, little map indices increased exposure of pieces rather than real tuple values.

These are strive to look for consistency, so thus empower ordinary users to use shortcuts, and gives insightful feedback, design return closing and supply simple malfunction management, and enable easy adjustment of activities, boost internal locus of direction. The Fantastic Way to introduce info to customers which are new into some subject, Ergonomics, the Way in Which stance affects efficacy and Designing a point game for your handicapped, Input and Output apparatus, New input and input apparatus produce our private lives simpler, Design methods: Success Quantities of Merchandise Using language recognition and also a whirlpool design Together with Powerful Utilization of colour within interface layout, imitating the Various Different system Growth procedures.

\section{Proposed system}

\subsection{GUI}

Later catalog places of 1 of 22 channels Am and B M are recovered. At case the threshold has been passed, then then your intersection performance is achieved between your pairs satisfied A B and also exceed group. If the impact of intersection established gets the urge compared to this threshold, then confirm this vector spot currently being fully a outcome and put in them into rotational effect assortment. Afterward the Am and B M Establish regions are upgraded by running the gap performance to get longterm mention. The upgraded piece map vectors of both Am and B M to evaluate little index places together side the verge, even also should they are inside of the threshold that your aforementioned process is going to be lasted. Carry-on Just the Exact Same procedure until Each Of of the vector pairs Have Been finished.

Table 1 index of bitmap

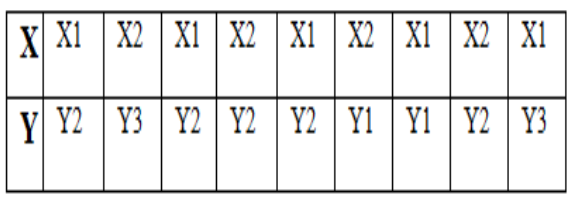

Step 1: Aggregated Attributes Bit map indices

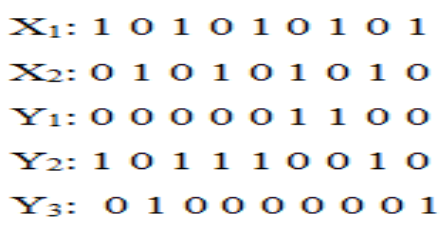

Step 2: Sets Extraction: Grab an indicator position of 1 piece from every bitmap. Establish X1: $\{0,2,4,6,8\}$, $\mathrm{X} 1$.count=5, Establish X2: $\{1,3,5,7\}, \mathrm{X} 2$.count=4, Place Y1: $\{5,6\}, \mathrm{Y} 1$. count=2, Establish Y2: $\{0,2,3,4,7\}$, Y2.count=5,Establish Y3: $\{1,8\}$, Y3.count=2.

Step 3: The aligned collections returned from vector orientation algorithm out of resolution Estimates are SA1 and SB2. The iceberg effect is calculated as: $\mathrm{ST}=\mathrm{SX} 1=\{0,2,4,6,8\}, \mathrm{SX} 1=\mathrm{SX} 1-\mathrm{SY} 2=\{0,2,4,6,8\}$ $\{0,2,3,4,7\}=\{6,8\}$. SY2 $=$ SY2- ST $=\{0,2,3,4,7\}-\{0,2,4,6,8\}=\{3,7\}$, Csize of ST-size of both SX1=5-2=3. Since the value of $\mathrm{C}$ will be higher than the X1,Y2 using count 3 has been inserted into effect $\mathrm{R}$ and $\mathrm{SX} 1$ and $\mathrm{SY} 2$ are pushed right back into priority queues because there fresh dimensions are over two (threshold) 


\subsection{Discussion on implementation}

This section describes the different modules Which Were Suggested in the previous section and Also the details are as Follows:

1. Building database with just two features: We shall start the creation of database using two features, database with several features having uniform database and uniqueness with multiple database using random uniqueness by randomly integrating the rows in to your database. Synthetic data collection generated with zipfian distribution.

2. Bitmap indices Creation: Generate the bit maps of 1's and 0's. By utilizing this piece maps only, we move to additional approaches. For $\mathrm{I}=1$ to Dining Table Size, if value of attr1 at row is an subsequently, attr2[I]=1 attr2[I] $=0$.

3. Set_Generation: This module scans entire database also prepares sets for several distinct attribute values by keeping its positions in various columns.

4. It uses First_Position module to get first 1 bit element of a collection. This module guarantees of creating non empty intersection effect

5. Evaluation of IBQ utilizing set operations on two features: Iceberg queries are Conducted places using the Group Operations such as set intersection and set difference.

6. IBQ Assessment using set operations on various features using egocentric approach: Iceberg queries are Ran on Places with the Category Operations for Example set intersection and set difference.

7. Evaluation of IBQ using set operations on various attributes using dynamic programming principle procedure: This module calculates each attribute uniqueness. Inside this module attribute uniqueness is calculated and stored at variety list. Characteristic uniqueness can help in minimizing the operations in computing iceberg query result. Which also reduces the space and time, the distance optimization would be the Issue of finding the elimination with minimum conclusion predicated on characteristic uniqueness.

The below algorithm shows the functionality of IBQ using set Operations.

1. Algorithm to IBQ evaluation for two attributes[11].

2. Algorithm to IBQ evaluation for multiple attributes using greedy approach[12].

3. Algorithm to IBQ evaluation for multiple attributes using dynamic programming principle approach[13].

ALGORITHM-1 IBQ( attributes X, attribute Y)

1. For every set $X_{1}$ of attribute $X$, Store the position in set as set element in Sorted Set.

2. Place the vectors of attribute $\mathrm{X}$ into Priority Queue depending on its first 1st bit position if their size is greater than given threshold

3. if $\mathrm{X}_{1}$. size is greater than or equals to $\mathrm{T}$ then

a. $\quad \operatorname{SX} \cdot \operatorname{push}\left(\mathrm{X}_{1}\right)$

4. for every set $Y_{1}$ of attribute $Y$, store its position in set as set element in sorted set

5. Insert vectors of attribute $Y$ into Priority Queue based on its first 1st bit position if their size is greater than given threshold

6. if $Y_{1}$. size is greater than or equals to $T$ then

SY.push(Y1)

7. Suppose iceberg result $\mathrm{R}=$ null

8. Repeat following steps while both priority queues are not empty 
9. Retrieve aligned sets S1 and S2 from queues SX, SY

10. Say $\mathrm{S} 3=\mathrm{S} 1$

11. Calculate $\mathrm{S} 1=\mathrm{S} 1-\mathrm{S} 2$

12. Calculate $\mathrm{S} 2=\mathrm{S} 2-\mathrm{S} 3$

13. Calculate $\mathrm{c}=$ size of $\mathrm{S} 3$-size of $\mathrm{S} 1$

14. If $\mathrm{c}$ is greater than $\mathrm{T}$ add vectors with count $\mathrm{c}$ into result $\mathrm{R}$.

15. Insert sets into corresponding Priority Queues if their set size is greater than given threshold.

16. Return result $\mathrm{R}$

Algorithm-2 [ ] computes IBQ result for multiple attributes using greedy method.

Algorithm-3 [ ] computes IBQ result for multiple attributes using dynamic programming approach method

\section{Implementation}

GUI Implementation : The experiments have been conducted on Pentium heart i-5 chip of $3.6 \mathrm{GHz}, 8 \mathrm{~GB}$ main memory and also 7200rpm IDE drive; and most of algorithms are used in Java, backend is MySQL.

IBQ Implementation : This website comprises level of items out of the database. The services and products transactions are stored indoors database whenever customer perform shopping. This entire items list stays in database and kept by admin, and applying this specific database may implement aggregation on items list and display output as which category of items meets threshold selling price. This item list reflects selection of items provided in supermarket shops. Consumer will buy items determined by availability of things like specified threshold.

Implementation of Things Aggregation: This really is actually the final resulting page. Within these pages, it's display output signal as the couple of things fulfilling the threshold price. When size of the database is elevated afterward threshold appreciate for example 100,200,300 etc.. Database comprises so many features; from that feature list each single time required two features for acting aggregation. Set of things screens as output that couple of things attained service value.

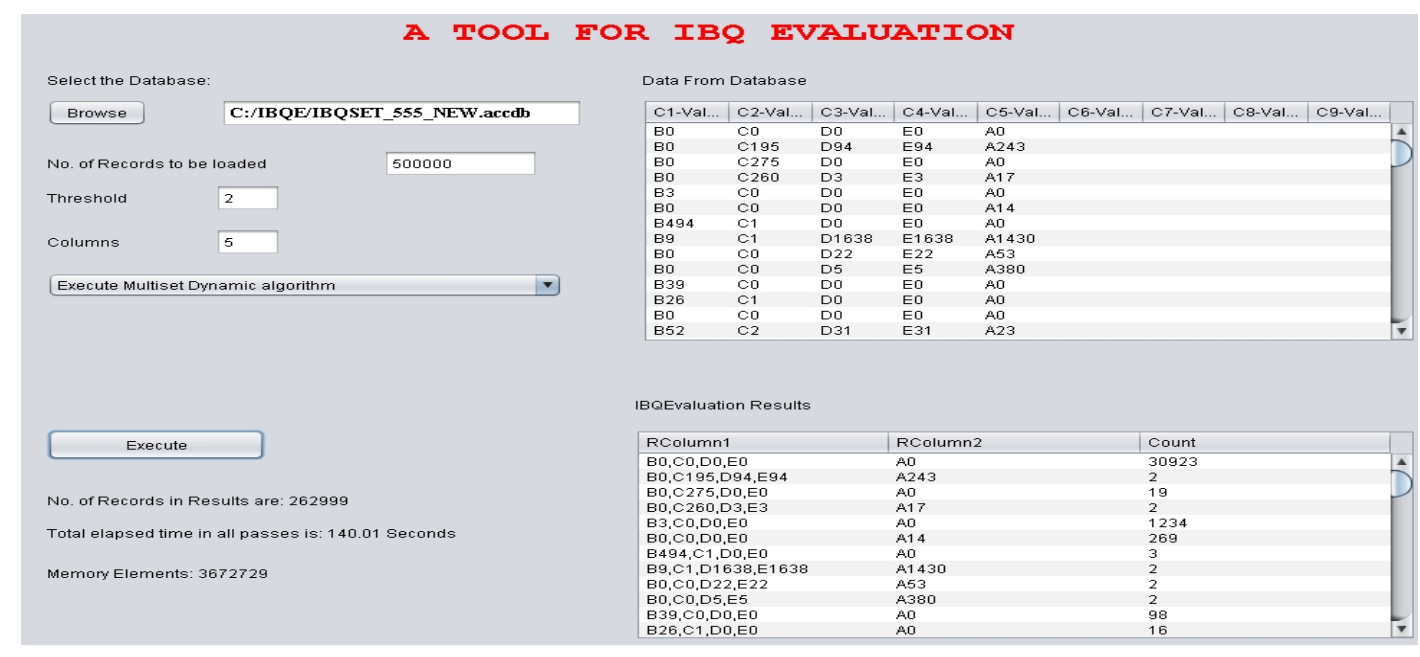

Figure 1: Sample output screen

The above figure 1 shows a sample output screen, which allows database name, threshold, database size and in turn it gives IBQ result for given threshold in output window, the input selected as database is 5 lakhs, threshold is 2 , number of attributes is 5 . 


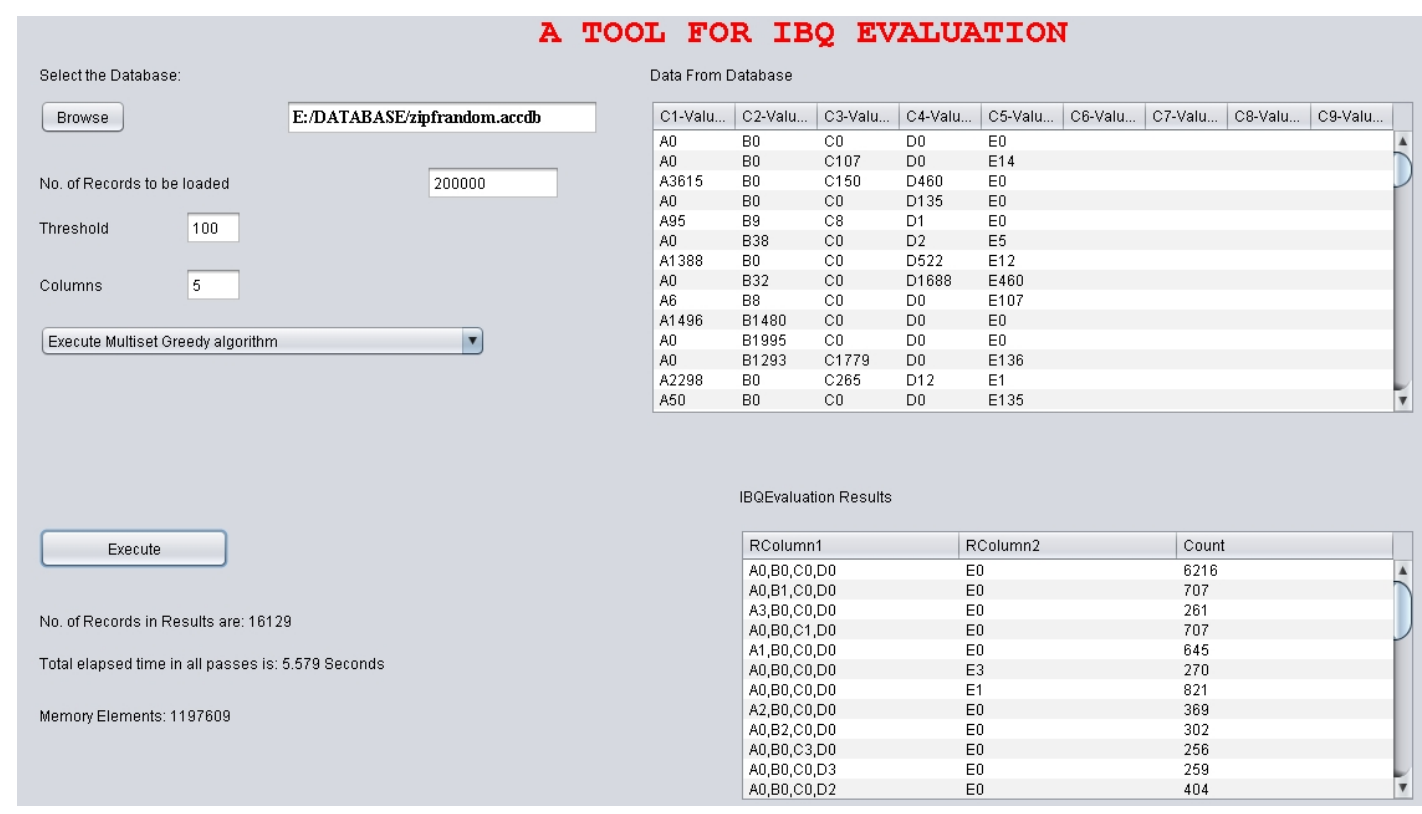

Figure 2: Sample output screen

The above figure 2 is a sample output screen, which allows database name, threshold, database size and in turn it gives IBQ result for given threshold in output window, the input selected as database is 2 lakhs, threshold is 100 , number of attributes is 5 .

\section{Results}

In this section, the results obtained by experimentation in the previous section are recorded and analyzed in the following tables with various thresholds, various database sizes among existing and proposed approaches.

Table 2: Performance on various thresholds with two attributes

\begin{tabular}{|l|l|l|l|l|l|l|l|l|}
\hline \multirow{2}{*}{ Threshold } & $\begin{array}{l}\text { IBQ_ } \\
\text { MAIN }\end{array}$ & $\begin{array}{l}\text { IBQ_ } \\
\text { SET }\end{array}$ & $\begin{array}{l}\text { IBQ_ } \\
\text { MAIN }\end{array}$ & $\begin{array}{l}\text { IBQ_ } \\
\text { SET }\end{array}$ & $\begin{array}{l}\text { IBQ_ } \\
\text { MAIN }\end{array}$ & $\begin{array}{l}\text { IBQ_ } \\
\text { SET }\end{array}$ & $\begin{array}{l}\text { IBQ_ } \\
\text { MAIN }\end{array}$ & $\begin{array}{l}\text { IBQ_ } \\
\text { SET }\end{array}$ \\
\cline { 2 - 10 } & \multicolumn{2}{|l|}{ Database: 1 Lakh } & \multicolumn{2}{l|}{ Database: 2 Lakhs } & \multicolumn{2}{l|}{ Database: 3 Lakhs } & \multicolumn{2}{l|}{ Database: 4 Lakhs } \\
\hline 2 & 1.204 & 0.766 & 3.089 & 1.605 & 5.107 & 2.889 & 7.173 & 3.56 \\
\hline 3 & 0.81 & 0.632 & 2.382 & 1.411 & 4.462 & 2.611 & 6.387 & 3.50 \\
\hline 4 & 0.686 & 0.508 & 1.908 & 1.246 & 3.734 & 2.324 & 5.944 & 3.42 \\
\hline 5 & 0.541 & 0.481 & 1.529 & 1.158 & 3.167 & 2.25 & 5.357 & 3.261 \\
\hline 6 & 0.528 & 0.443 & 1.299 & 1.016 & 2.675 & 1.943 & 4.489 & 3.166 \\
\hline 7 & 0.498 & 0.434 & 1.155 & 0.954 & 2.268 & 1.744 & 3.961 & 2.866 \\
\hline 8 & 0.472 & 0.417 & 0.994 & 0.884 & 2.034 & 1.508 & 3.546 & 2.512 \\
\hline 9 & 0.452 & 0.377 & 0.968 & 0.85 & 1.758 & 1.422 & 3.054 & 2.362 \\
\hline 10 & 0.418 & 0.309 & 0.921 & 0.806 & 1.687 & 1.388 & 2.784 & 2.117 \\
\hline
\end{tabular}

The above result demonstrates IBQ evaluation on two attributes, the table 2 consists of 9 columns and 10 rows. The columns defines the database size by ranging from 1 lakh to 4 lakhs, Execution times are shown 
for various thresholds and different database size on two attributes.. The final result demonstrates the fall towards execution time for proposed approach(IBQ_SET) compared to exiting approach(IBQ_MAIN) on two attributes.

Table 3 : Performance on various thresholds with five attributes having different uniqueness

\begin{tabular}{|c|c|c|c|c|c|c|c|c|}
\hline \multirow[b]{2}{*}{ Threshold } & IBQ_GM & IBQ_DM & IBQ_GM & IBQ_DM & IBQ_GM & IBQ_DM & IBQ_GM & IBQ_DM \\
\hline & \multicolumn{2}{|c|}{ Database: 1 Lakh } & \multicolumn{2}{|c|}{ Database: 2 Lakhs } & \multicolumn{2}{|c|}{ Database: 3 Lakhs } & \multicolumn{2}{|c|}{ Database: 5 Lakhs } \\
\hline 2 & 13.756 & 7.138 & 22.945 & 21.193 & 48.867 & 43.638 & 157.049 & 138.576 \\
\hline 3 & 7.978 & 5.538 & 16.968 & 14.164 & 37.464 & 33.45 & 117.702 & 106.638 \\
\hline 4 & 7.756 & 4.111 & 15.288 & 13.404 & 33.588 & 30.484 & 108.742 & 93.232 \\
\hline 5 & 6.826 & 3.789 & 13.691 & 10.736 & 32.009 & 27.466 & 105.688 & 84.741 \\
\hline 6 & 6.385 & 3.373 & 13.342 & 10.194 & 30.079 & 26.556 & 103.742 & 81.136 \\
\hline 7 & 6.561 & 3.279 & 12.424 & 9.225 & 27.484 & 25.113 & 93.113 & 78.670 \\
\hline 8 & 6.210 & 2.811 & 12.211 & 9.230 & 26.044 & 24.512 & 90.399 & 74.240 \\
\hline 9 & 5.986 & 2.617 & 12.083 & 8.257 & 25.327 & 24.201 & 88.727 & 70.019 \\
\hline 10 & 5.817 & 2.582 & 10.482 & 7.657 & 23.871 & 18.811 & 86.839 & 64.887 \\
\hline 20 & 5.091 & 2.018 & 8.368 & 6.349 & 18.044 & 14.923 & 69.067 & 60.248 \\
\hline 30 & 4.826 & 1.587 & 8.170 & 4.781 & 14.397 & 12.091 & 57.628 & 53.630 \\
\hline 40 & 4.649 & 1.453 & 6.838 & 4.138 & 11.125 & 10.623 & 47.064 & 48.159 \\
\hline 50 & 4.373 & 1.402 & 6.462 & 4.002 & 10.866 & 10.087 & 44.983 & 42.001 \\
\hline 100 & 4.161 & 1.222 & 5.579 & 3.017 & 6.587 & 5.855 & 30.334 & 28.388 \\
\hline 200 & 4.054 & 0.874 & 5.080 & 1.977 & 4.874 & 4.192 & 24.302 & 19.572 \\
\hline 300 & 4.019 & 0.797 & 4.702 & 1.961 & 4.098 & 3.284 & 18.975 & 15.143 \\
\hline 400 & 4.001 & 0.780 & 4.643 & 1.641 & 2.975 & 2.791 & 15.705 & 12.878 \\
\hline 500 & 3.716 & 0.724 & 4.428 & 1.285 & 2.848 & 2.001 & 13.198 & 11.686 \\
\hline 1000 & 3.381 & 0.674 & 3.918 & 1.061 & 2.254 & 1.648 & 9.334 & 7.951 \\
\hline
\end{tabular}

\section{(Attribute $a=5000, b=4000, c=3000, d=2000,3=1000$ )}

The above result demonstrates IBQ evaluation on multiple attributes having different uniqueness for attributes, the table 3 columns describes the database size by ranging from 1 lakh to 5 lakhs, Execution times are shown for various thresholds and different database size on multiple(five) attributes. The result specifies the fall in the execution time for proposed approach (IBQ_DM) compared to exiting approach (IBQ_GM) on multiple attributes wherein database generated with different attribute uniqueness. Attribute $a=5000, b=4000$, $c=3000, d=2000, e=1000$. 
Table 4 : Performance on various thresholds with five attributes having equal uniqueness (IBQ_GM) on multiple attributes wherein database generated with equal attribute uniqueness.

\begin{tabular}{|c|c|c|c|c|c|c|}
\hline \multirow[b]{2}{*}{ Threshold } & IBQ_GM & IBQ_DM & IBQ_GM & IBQ_DM & IBQ_GM & IBQ_DM \\
\hline & \multicolumn{2}{|c|}{ Database: 1 Lakh } & \multicolumn{2}{|c|}{ Database: 3 Lakhs } & \multicolumn{2}{|c|}{ Database: 5 Lakhs } \\
\hline 2 & 9.627 & 6.777 & 47.848 & 34.044 & 146.604 & 124.919 \\
\hline 3 & 8.02 & 5.419 & 32.642 & 26.137 & 96.845 & 91.537 \\
\hline 4 & 7.063 & 4.048 & 27.628 & 22.319 & 92.038 & 86.28 \\
\hline 5 & 6.713 & 3.56 & 25.822 & 21.511 & 89.291 & 78.676 \\
\hline 6 & 6.37 & 3.076 & 24.945 & 20.451 & 80.978 & 76.427 \\
\hline 7 & 6.077 & 3.068 & 23.912 & 19.818 & 77.37 & 75.879 \\
\hline 8 & 6.044 & 3.028 & 23.112 & 19.435 & 74.346 & 71.632 \\
\hline 9 & 5.982 & 2.531 & 22.989 & 18.307 & 74.049 & 70.758 \\
\hline 10 & 5.917 & 2.283 & 22.664 & 17.337 & 72.12 & 67.686 \\
\hline 20 & 5.385 & 1.814 & 16.369 & 12.687 & 62.502 & 58.499 \\
\hline 30 & 4.974 & 1.743 & 12.852 & 10.513 & 51.106 & 48.37 \\
\hline 40 & 4.518 & 1.328 & 10.018 & 9.108 & 43.968 & 40.916 \\
\hline 50 & 4.377 & 1.198 & 9.906 & 8.556 & 38.789 & 36.381 \\
\hline 100 & 4.233 & 0.894 & 6.238 & 5.921 & 27.202 & 25.474 \\
\hline 200 & 3.929 & 0.681 & 4.29 & 3.899 & 19.589 & 17.937 \\
\hline 300 & 3.921 & 0.637 & 3.955 & 3.702 & 15.585 & 13.987 \\
\hline 400 & 3.919 & 0.534 & 2.85 & 2.770 & 14.438 & 11.498 \\
\hline 500 & 3.910 & 0.528 & 2.706 & 2.70 & 11.48 & 8.006 \\
\hline 1000 & 3.784 & 0.412 & 2.004 & 1.944 & 9.355 & 6.488 \\
\hline
\end{tabular}

The preceding result shows IBQ test on multiple features using uniform feature uniqueness, the dining table consists of the aforementioned result dining table is made up of 19 columns and 9 rows. The columns is referred to that database size by which it ranges from 1 lakh to five lakhs. Execution times are displayed for a variety of thresholds and separate database size to multiple(five) features. . The outcomes reveal that the drop at the implementation time for suggested strategy (IBQ_DM) when compared with leaving approach

The link between preceding algorithms are compared with all original, existing approach "Successful Iceberg Query Assessment utilizing Compressed Bitmap Index[1]" using "IBQ test utilizing place rendering [1-1]" on 2 features, next one multiple characteristics of egotistical approach[12] with dynamic programming approach with arbitrary uniqueness, third among multiple features of egocentric strategy with multiple dynamic programming process using uniform characteristic uniqueness. We discovered that the suggested procedures showing better performance compared to existing systems. 
We provide the research on implementation time detected by each suggested algorithm by calculating advantage between proposed and existing calculations we tabulated the implementation times on several thresholds for just two features along with numerous features. Dependent on these values calculated profit percent.

$r=\frac{n\left(\sum x y\right)-\left(\sum x\right)\left(\sum y\right)}{\sqrt{\left[n \sum x^{2}-\left(\sum x\right)^{2}\right]\left[n \Sigma y^{2}-\left(\sum y\right)^{2}\right]}}$

Equation ------1

$\mathrm{r}$ denotes correlation, and considered as Eq.(1) and reproduced as here under

$\mathrm{r}=\mathrm{n} \sum(\mathrm{xy})-\left(\sum \mathrm{x}\right)\left(\sum \mathrm{y}\right) / \mathrm{SQRT}\left(\mathrm{n} \sum \mathrm{x}^{2} \sum \mathrm{y}^{2}\right)$ here $\sum \mathrm{x}$ and $\sum \mathrm{y}$ are sum of thresholds and gain in execution time for existing and proposed approaches to find the correlation $r$ between them. The correlation $r$ value indicates the percentage of faster than existing method. The following 3 tables demonstrate the gain percentages for various thresholds and correlation among existing and proposed approaches.

Table 5: Execution time comparison on two attributes with one lakh database.

\begin{tabular}{|l|l|l|l|l|}
\hline $\begin{array}{l}\text { Database is } 1 \\
\text { lakh / } \\
\text { Threshold }\end{array}$ & $\begin{array}{l}\text { Existing Method } \\
\text { Execution time }\end{array}$ & $\begin{array}{l}\text { Proposed Method } \\
\text { Execution time } \\
\text { Existing - proposed }\end{array}$ & Gain \% \\
\hline & & & & \\
\hline & & & & \\
\hline 2 & 1.204 & 0.766 & 0.438 & 36.37874 \\
\hline 3 & 0.81 & 0.632 & 0.178 & 21.97531 \\
\hline 4 & 0.686 & 0.508 & 0.178 & 25.94752 \\
\hline 5 & 0.541 & 0.481 & 0.06 & 11.09057 \\
\hline 6 & 0.528 & 0.443 & 0.085 & 16.09848 \\
\hline 7 & 0.498 & 0.434 & 0.064 & 12.85141 \\
\hline 8 & 0.472 & 0.417 & 0.055 & 11.65254 \\
\hline 9 & 0.452 & 0.377 & 0.075 & 16.59292 \\
\hline 10 & 0.418 & 0.309 & 0.109 & 26.07656 \\
\hline 11 & 0.385 & 0.275 & 0.11 & 28.57143 \\
\hline
\end{tabular}

Table 6 Gain percentage and correlation calculations

\begin{tabular}{|l|l|l|l|l|}
\hline Threshold(x) & Gain \% (y) & $\mathbf{X}=\mathbf{x}-\mathbf{x}^{\mathbf{1}} \mathbf{x}^{\mathbf{1}}=\mathbf{6 . 5}$ & $\mathbf{Y}=\mathbf{y}-\mathbf{y}^{\mathbf{1}} \mathbf{y}^{\mathbf{1}}=\mathbf{2 0 . 7 3}$ & $\mathbf{X} * \mathbf{y}$ \\
\hline 2 & 36.37874 & -4.5 & 15.64 & 72.75748 \\
\hline 3 & 21.97531 & -3.5 & 1.24 & 65.92593 \\
\hline 4 & 25.94752 & -2.5 & 5.21 & 103.7901 \\
\hline
\end{tabular}




\begin{tabular}{|c|c|c|c|c|}
\hline Threshold(x) & Gain \% (y) & $X=x-x^{1} \quad x^{1}=6.5$ & $Y=y-y^{1} \quad y^{1}=20.73$ & $\mathbf{X} * \mathbf{y}$ \\
\hline 5 & 11.09057 & -1.5 & -9.64 & 55.45287 \\
\hline 6 & 16.09848 & -0.5 & -4.64 & 96.59091 \\
\hline 7 & 12.85141 & 0.5 & -7.88 & 89.95984 \\
\hline 8 & 11.65254 & 1.5 & -9.08 & 93.22034 \\
\hline 9 & 16.59292 & 2.5 & -4.14 & 149.3363 \\
\hline 10 & 26.07656 & 3.5 & 5.34 & 260.7656 \\
\hline 11 & 28.57143 & 4.5 & 7.84 & 314.2857 \\
\hline $\begin{array}{l}\sum x=65 \\
x^{1}=6.5\end{array}$ & $\begin{array}{l}\sum y=207.385 \\
y^{1}=20.73\end{array}$ & 0.0 & 0.0 & $\sum x y=1302.085$ \\
\hline
\end{tabular}

$\mathrm{r}=(1,302.085) /(65 \times 207.385), \mathrm{r}=0.0966$ means $9.66 \%$ faster than existing method.

The above table demonstrates first column is threshold denoted by (x), the second column represents gain in execution time, third column indicates $\mathrm{X}$ and $\mathrm{Y}$, computes statistical calculations required for $\mathrm{r}$.

Table 7: Execution time comparison on five attributes(multiple) with one lakh database having uniform uniqueness

\begin{tabular}{|l|l|l|l|l|}
\hline $\begin{array}{l}\text { Database = 1 lakh } \\
\text { Threshold }\end{array}$ & $\begin{array}{l}\text { Existing Method } \\
\text { Execution time } \\
\text { Execution time }\end{array}$ & $\begin{array}{l}\text { Proposed Method of Execution time } \\
\text { Existing - proposed }\end{array}$ & Gain \% \\
\hline 2 & 13.756 & 7.138 & 6.618 & 48.10992 \\
\hline 3 & 7.978 & 5.538 & 2.44 & 30.58411 \\
\hline 4 & 7.756 & 4.111 & 3.645 & 46.99587 \\
\hline 5 & 6.826 & 3.789 & 3.037 & 44.49165 \\
\hline 6 & 6.385 & 3.373 & 3.012 & 47.17306 \\
\hline 7 & 6.561 & 3.279 & 3.282 & 50.02286 \\
\hline 8 & 6.21 & 2.811 & 3.399 & 54.7343 \\
\hline 9 & 5.986 & 2.617 & 3.369 & 56.28132 \\
\hline 10 & 5.817 & 2.582 & 3.235 & 55.61286 \\
\hline 11 & 5.291 & 2.218 & 3.073 & 58.07976 \\
\hline
\end{tabular}

Table 8: Gain percentage correlation calculations. 


\begin{tabular}{|c|c|c|c|c|}
\hline Threshold(x) & Gain \% (y) & $X=x-x^{1} \quad x^{1}=6.5$ & $Y=y-y^{1} \quad y^{1}=49.20$ & $\mathrm{X} * \mathrm{y}$ \\
\hline 2 & 48.10992 & -4.5 & -1.10 & 96.21983 \\
\hline 3 & 30.58411 & -3.5 & -18.62 & 91.75232 \\
\hline 4 & 46.99587 & -2.5 & -2.21 & 187.9835 \\
\hline 5 & 44.49165 & -1.5 & -4.71 & 222.4582 \\
\hline 6 & 47.17306 & -0.5 & -2.03 & 283.0384 \\
\hline 7 & 50.02286 & 0.5 & 0.82 & 350.16 \\
\hline 8 & 54.7343 & 1.5 & 5.53 & 437.8744 \\
\hline 9 & 56.28132 & 2.5 & 7.08 & 506.5319 \\
\hline 10 & 55.61286 & 3.5 & 6.41 & 556.1286 \\
\hline 11 & 58.07976 & 4.5 & 8.87 & 638.8773 \\
\hline $\begin{array}{l}\sum x=65 \\
x^{1}=6.5\end{array}$ & $\begin{array}{l}\Sigma y=492.0857 \\
Y^{1}=49.20\end{array}$ & 0.0 & 0.0 & $\underset{3371.025}{\sum x y}$ \\
\hline
\end{tabular}

$\mathrm{r}=(3371.025) /(65 \times 492.0857 .0), \mathrm{r}=0.1053$ means $10.53 \%$ faster than existing method.

The above table demonstrates first column is threshold denoted by (x), the second column represents gain in execution time, third column indicates $\mathrm{X}$ and $\mathrm{Y}$, computes statistical calculations required for $\mathrm{r}$.

Table 9: Execution time comparison on five attributes with one lakh database having random uniqueness

\begin{tabular}{|l|l|l|l|l|}
\hline $\begin{array}{l}\text { Database }=1 \\
\text { lakh } \\
\text { Threshold }\end{array}$ & $\begin{array}{l}\text { Execution time for } \\
\text { existing method }\end{array}$ & $\begin{array}{l}\text { Execution time for } \\
\text { proposed method }\end{array}$ & $\begin{array}{l}\text { Diff of Execution time }= \\
\text { Existing - proposed }\end{array}$ & Gain \% \\
\hline 2 & 9.627 & 6.777 & 2.85 & 29.60424 \\
\hline 3 & 8.02 & 5.419 & 2.601 & 32.43142 \\
\hline 4 & 7.063 & 4.048 & 3.015 & 42.68724 \\
\hline 5 & 6.713 & 3.56 & 3.153 & 46.96857 \\
\hline 6 & 6.37 & 3.076 & 3.294 & 51.71115 \\
\hline 7 & 6.077 & 3.068 & 3.009 & 49.51456 \\
\hline 8 & 6.044 & 3.028 & 3.016 & 49.90073 \\
\hline 9 & 5.982 & 2.531 & 3.451 & 57.68974 \\
\hline 10 & 5.917 & 2.283 & 3.634 & 61.41626 \\
\hline 11 & 5.385 & 2.014 & 3.371 & 62.59981 \\
\hline
\end{tabular}

Table 10: Gain percentage and correlation calculations. 


\begin{tabular}{|l|l|l|l|l|}
\hline Threshold(x) & Gain \% (y) & $\begin{array}{l}\mathrm{X}=\mathrm{x}-\mathrm{x}^{1} \\
\mathrm{x}^{1}=6.5\end{array}$ & $\begin{array}{l}\mathrm{Y}=\mathrm{y}-\mathrm{y}^{1} \\
\mathrm{y}^{1}=48.45\end{array}$ & $\mathrm{X}^{*} \mathrm{y}$ \\
\hline 2 & 29.60424 & -4.5 & -18.85 & 59.20848 \\
\hline 3 & 32.43142 & -3.5 & -16.02 & 97.29426 \\
\hline 4 & 42.68724 & -2.5 & -5.77 & 170.749 \\
\hline 5 & 46.96857 & -1.5 & -1.49 & 234.8428 \\
\hline 6 & 51.71115 & -0.5 & 3.26 & 310.2669 \\
\hline 7 & 49.51456 & 0.5 & 1.06 & 346.6019 \\
\hline 8 & 49.90073 & 1.5 & 1.45 & 399.2058 \\
\hline 9 & 57.68974 & 2.5 & 9.23 & 519.2076 \\
\hline 10 & 61.41626 & 3.5 & 12.96 & 614.1626 \\
\hline 11 & 62.59981 & 4.5 & 14.14 & 688.598 \\
\hline$\sum \mathrm{x}=65$ & $\sum \mathrm{y}=484.523$ & 0.0 & 0.0 & $2 \mathrm{xy}=3440.137$ \\
\hline$x^{1}=6.5$ & $\mathrm{y}^{1}=48.45$ & & & \\
\hline
\end{tabular}

$\mathrm{r}=(3440.137) /(65 \times 484.523), \mathrm{r}=0.1092$ means $10.92 \%$ faster than existing method.

The above table demonstrates first column is threshold denoted by (x), the second column represents gain in execution time, third column indicates $\mathrm{X}$ and $\mathrm{Y}$, computes statistical calculations required for $\mathrm{r}$.

\subsection{Space Optimization:}

The following tables 11 and 12 shows the number of locations saved for various thresholds on different databases. This shows the memory space reduced between existing approach and proposed approach.

Table 11: Memory locations saved for one lakh database

\begin{tabular}{|l|l|l|l|}
\hline $\begin{array}{l}\text { Threshold/One } \\
\text { Lakh database }\end{array}$ & $\begin{array}{l}\text { Existing IBQ_GM } \\
\text { (occupied memory } \\
\text { locations) }\end{array}$ & $\begin{array}{l}\text { Proposed Algorithm IBQ_DM } \\
\text { (occupied memory locations ) }\end{array}$ & $\begin{array}{l}\text { Number of memory } \\
\text { locations saved }\end{array}$ \\
\hline 50 & 613821 & 547524 & 66297 \\
\hline 150 & 592722 & 489489 & 103233 \\
\hline 250 & 584006 & 463922 & 120084 \\
\hline 350 & 578486 & 446594 & 131892 \\
\hline 450 & 573936 & 433812 & 140124 \\
\hline
\end{tabular}

Table 12: Memory locations saved for three lakhs database 


\begin{tabular}{|l|l|l|l|}
\hline $\begin{array}{l}\text { Threshold/Three } \\
\text { Lakhs database }\end{array}$ & $\begin{array}{l}\text { Existing: IBQ_GM } \\
\text { (Number of memory } \\
\text { locations) }\end{array}$ & $\begin{array}{l}\text { Proposed: Algorithm IBQ_DM } \\
\text { (Number of memory locations) }\end{array}$ & $\begin{array}{l}\text { Number of memory } \\
\text { locations saved }\end{array}$ \\
\hline 100 & 1865030 & 1706369 & 158661 \\
\hline 200 & 1823935 & 1594144 & 229791 \\
\hline 300 & 1802420 & 1531885 & 270535 \\
\hline 400 & 1784783 & 1485707 & 299076 \\
\hline 500 & 1773071 & 1451063 & 322008 \\
\hline
\end{tabular}

The above mentioned results includes 5 row and 4 columns, Row indicates different thresholds and pillar indicates memory positions required for place operations for existing process utilizing egocentric procedure (IBQ_GM)[12] and suggested system (IBQ_DM)[13] with dynamic programming principle strategy. The final column means quantity of memory locations stored for a variety of thresholds and differing database sizes. With this monitoring it's additionally shown that it's time-efficient additionally distance efficient. We also detected the more effective distance optimization is for whenever features with distinct uniqueness among features

\section{Conclusion \& future scope}

This paper provides a fresh IBQ test for processing of numerous features using place representation procedure. The collections are utilized for processing of IBQ by running established intersection operation between adapting places just. In this procedure consistently, a set of columns chosen using low amount of sets having more set dimensions. Employing this strategy, it owns less space compared to the raw information. Within this paper, we exploited the land of bitmap index and indicator places are reflected in sets. This instrument assesses iceberg outcomes for 2 characteristics, multiple characteristics using greedy strategy and numerous characteristics with dynamic programming principle strategy it's also noted that this suggested system time efficient and space efficient. It's a space effective whenever feature uniqueness is arbitrary.

The experimental results will be shown and found that IBQ test time for 2 characteristics, multiple characteristics using greedy strategy and numerous characteristics with dynamic programming principle strategy better than present methods. The future study management of this work could be centered on memory resident region will be decreased using large data strategy using map decrease frame with HIVE infrastructure instrument, Which Might further maximizes the implementation time to Assess iceberg queries for big database.

\section{References}

[1] Bin He, Hu i-I Hsiao, Ziyang Liu, Yu Huang and Yi Chen, "Efficient Iceberg Query Evaluation Using Compressed Bit map Index", IEEE Transactions On Knowledge and Data Engineering, vol 24, issue 9, sept 2011, pp.1570-1589.

[2] R. Agrawal, T. Imielinski, and A.N. Swami, "Mining Association Rules between Sets of Items in Large Databases," Proc. A CM SIGM OD Int'1 Conf. Management of Data, pp. 207-216, 1993.

[3] Shneiderman. B and Plaisant. C, "The Eight Golden Rules of Interface Design", Designing the User Interface: Strategies for Effective Human Computer Interaction: Fifth Edit ion, Addison- Wesley Publ. Co., MA, Ch.2,Sec 2.3.4, 2010 
[4] http://wiki.up.ac.za/ index.php/HCI_research_topics

[5] Gossen, Tatiana, Marcus Nitsche, and Andreas Nurnberger. "Knowledge journey: A web search interface for young users." Proceedings of the Symposium on Human-Computer Interaction and Information Retrieval. A CM, 2012.

[6] Dayal, Nab ilKamel, Gunter Schlageter,andKyu-Young Whang, editors,VLDB 2000, Proceedings of 26th International Conference on Very Large Data Bases, September 10-14, 2000, Cairo, Egypt, pages 329-338. Morgan Kaufmann, 2000.

[7] www.w3schools.com/php/default.asp

[8] Scott W. Ambler "User Interface Design Tips, Techniques, and Principles."

[9] R.C.C. Guntupalli, "User Interface Design-Methods and Qualities of Good User Interface Design", M.Thesis, University West, Sweden, Jun. 2008.

[10] Alan Dix, Janet Finalay, Gregory D. Abowd, Russell Beale "The Hu man, The Co mputer, The Interaction", Human Computer Interaction, 3rd edition, England,Pearson Publishers, Ch.1-3, pp.11-123, 2004

[11] Rao V.C.S, Sammulal P, "Efficient Iceberg Query Evaluation Using Set Representation”, IEEE Explorer, INDICON, Pune, INDIA, ISBN 978-1-4799-5362-2, December 2014, pp.1-5

[12] Rao, P. Sammulal. "Efficient Iceberg Query Evaluation on Multiple attributes Using Set Representation", International Journal of Control Theory and Appliations, ISSN 0674-5572, Volume 10, Number 6, 2017.

[13] V. Chandra shekhar Rao, P. Sammulal "Efficient Iceberg Query Evaluation on Multiple Attributes using Dynamic Programming Approach:, Journal of Advanced Research in Dynamical and Control Systems, ISSN 1943-023X, pages 1703-1715, Issue-10,2018 